\title{
KERR BLACK HOLES AND JETS
}

\author{
Arun Kenath and Samartha C. $A^{*}$ \\ Chandrashekaran**
}

\begin{abstract}
The work presented in this paper is part of our project titled "Aspects of Black Hole Energetics" during the fourth semester of M.Sc. (physics) under the guidance of Prof. C. Sivaram, IIA. We are very thankful to Prof. C. Sivaram for his guidance through out the project and also to Prof. K. A. Chandrashekar, H.O.D, P.G Dept of Physics, Christ College, for the support and encouragement.
\end{abstract}

\section{Black holes}

A black hole is basically a region of space-time that has so much mass concentrated in it that there is no way for a nearby object to escape its gravitational pull. Since our best theory of gravity at the moment is Einstein's general theory of relativity, we have to delve into some results of this theory to understand black holes in detail. The basic parameter associated with a black hole is its Schwarzschild radius.

The solution to the Einstein equations for the space-time around a planet or star of mass $M$ is called the Schwarzschild metric [1], given as:

$$
d r^{2}=\left(1-\frac{2 M G}{c^{2} r}\right) d t^{2}-\left(1-\frac{2 M G}{c^{2} r}\right)^{-1} d r^{2}-r^{2} d \theta^{2}-r^{2} \sin ^{2} \theta d \phi^{2} .
$$

* Former MSc Students, Department of Physics, Christ College, Bangalore.

** Prof and Heod of PG Department of Physics, Christ College. 
The radius at which the metric becomes singular is the Schwarzschild radius and this is given by,

$$
R_{s}=\frac{2 G M}{c^{2}}
$$

The surface at this radius is called the event horizon. The above solution corresponds to a case where the angular momentum associated with the black hole is zero. In this paper we shall consider a spinning black hole that is the Kerr black holes.

\section{Kerr black holes}

The exact solution of the Einstein field equations outside a spinning sphere of mass $M$ and angular momentum $J$ is given by the Kerr solution [6] as,

$$
\begin{aligned}
d r^{2}= & -\left(1-\frac{2 M r}{\Sigma}\right) d t^{2}-\frac{4 a M r \sin ^{2} \theta}{\Sigma} d t d \phi+\frac{\Sigma}{\Delta} d r^{2} \\
& +\sum d \theta^{2}+\left(r^{2}+a^{2}+\frac{2 M r a^{2} \sin ^{2} \theta}{\Sigma}\right) \sin ^{2} \theta d \phi^{2}
\end{aligned}
$$

where the black hole is rotating in the $\phi$ direction and

$$
a=\frac{J}{M} ; \Delta=r^{2}-2 M r+a^{2} ; \Sigma=r^{2}+a^{2} \cos ^{2} \theta
$$

A Kerr black hole adds another feature to the anatomy of the black hole, an ergosphere. The ergosphere resides in an ellipsoidal region outside the outer event horizon. The ergosphere represents the last stable orbit, and the outer boundary is called the static limit.

Yet another distinguishing feature of the Kerr black hole is that, since it rotates the $0-D$ point that is the singularity in the black hole is spun into a ring of zero thickness. Interesting theoretical physics can take place around this ring singularity. One consequence is that nothing can actually fall into it unless it approaches along a trajectory along the ring's side. Any other angle and the ring actually produce an antigravity field that repels matter. 


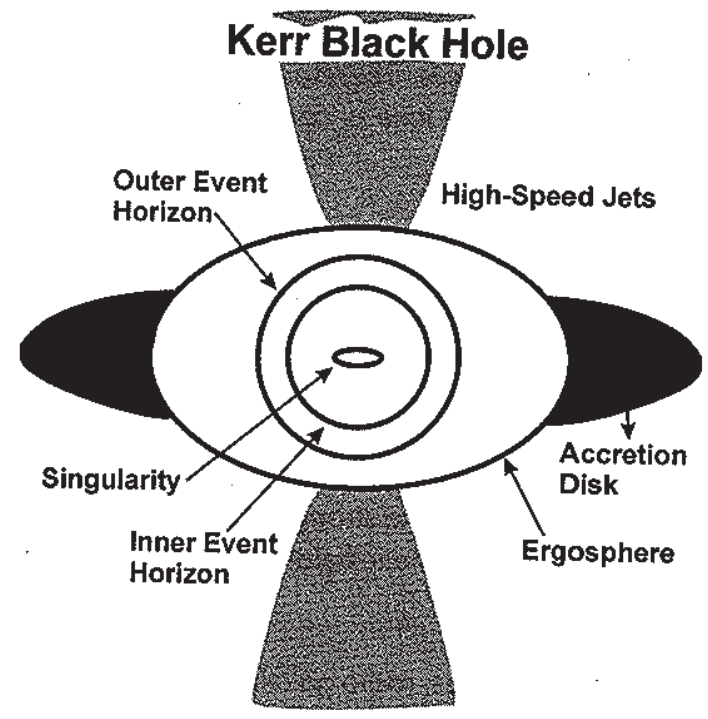

Fig 1.1: Kerr black hole [7]

The horizon for the Kerr black hole is given by,

$$
r=m \pm \sqrt{m^{2}-a^{2}}
$$

Here $m$ is the geometric mass and $a$ is the geometric angular momentum. From the condition that $r$ should be real, the limiting case is given by, $m=a$.

$$
\text { i.e. } \frac{G M}{c^{2}}=\frac{J_{M A X}}{M c^{2}}
$$

From this, the maximum angular momentum is given by, $J_{\max }=\frac{M^{2} G}{c}$. This is the maximum allowed angular momentum associated with a Kerr black hole.

For a $10 \mathrm{M}_{\theta}$ black hole, the maximum angular momentum allowed as per the above expression is of the order of, $J_{\text {MAx }} \approx 10^{43} \mathrm{kgm}^{2} / \mathrm{s}$.

If we assume a $10 M_{\theta}$ star of radius $1.5 R_{\theta}$ with an equatorial velocity of $v=300 \mathrm{~km} / \mathrm{s}$, then its angular momentum is given by, $J=m v r \approx 10^{44} \mathrm{kgm}^{2} / \mathrm{s}$. 
We see that this angular momentum is larger than the maximum allowed angular momentum for a rotating black hole. Therefore, for such early type stars to end up as black holes, they will have to lose a considerable amount of their angular momentum.

\section{Jets from a Black Hole}

The effect of spin of the black hole produces a very interesting phenomenon. A long-standing puzzle has been how systems such as radio galaxies and quasars launch powerful bi-directional jets. The current consensus is that the process involves a supermassive black hole that is being fed with magnetized gas through an orbiting accretion disk. The combination of the strong gravity of the black hole, the rotation in the in falling matter, and the magnetic field are believed to be the key ingredients to jet creation.

Objects as diverse as $X$-ray binaries, radio galaxies, quasars, and even our Galactic centre, are powered by the gravitational energy released when surrounding gas is sucked into the black hole sitting in their cores, a process astronomers call accretion.

Apart from copious radiation, one of the manifestations of this accretion energy release is the production of so-called jets, collimated beams of matter that are expelled from the innermost regions of accretion disks. These jets shine particularly brightly at radio frequencies.

An accretion disk is matter that is drawn to the black hole. In rotating black holes, the matter forms a disk due to the mechanical forces present. In an Schwarzschild black hole, the matter would be drawn in equally from all directions, and thus would form an omni-directional accretion cloud rather than disk. Jets form in Kerr black holes that have an accretion disk. The matter is funnelled into a disk-shaped torus by the black hole's spin and magnetic fields, but in the very narrow regions over the black hole's poles, matter can be energized to extremely high temperatures and speeds, escaping the black hole in the form of high-speed jets [9].

The jet is powered by both the energy of accretion in the disk and from the rotational energy of the black hole. It is the rotating black hole; however, that provides most of the energy.

We have already seen the expression for the maximum angular momentum for a black hole, which is given by, $J_{\max }=\frac{M^{2} G}{c}$ 
From the classical expression for the angular momentum associated with a jet of length l, assuming the particles to be travelling at near speed of light, the expression becomes $J=m c l$.

Considering a conical jet with base radius $r$ and density $r$, the mass of the jet is given by, $m=\frac{1}{3} \pi r^{2} \rho$

Then the angular momentum becomes $J=\frac{1}{3} \pi l^{2} r^{2} c \rho$

From the geometry of the jet, we can relate the length of the jet to the radius $r$ as $r=1 \tan 5^{\circ}$. Here we have assumed the small opening angle of the jet to be $5^{\circ}$.

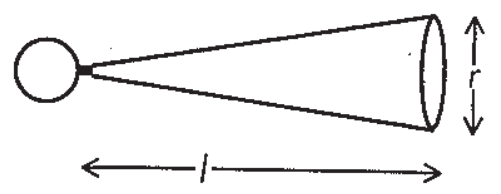

Fig 1.2: Geometry of the jet

Assuming the number density of the jet to be of the order of $10^{3}$, which is consistent with observations, the length of the jet is given by

$$
I=\left(\frac{3 G M^{2}}{\pi \rho c^{2}(\tan 5)^{2}}\right)^{1 / 4}
$$

For the billion solar mass black holes, it works out to $\mathrm{I}=6 \times 10^{19} \mathrm{~m}=2 \mathrm{kpc}$.

The scaling of the length of the jet with the mass of the central black hole is given by

$$
\begin{aligned}
& 1=\left(\frac{3 G}{\pi \rho c^{2}(\tan 5)^{2}}\right)^{1 / 4} M^{1 / 2} \\
& 1 \approx 0.5 \sqrt{M}
\end{aligned}
$$


The figure below shows the jet emitted out of the accretion disc surrounding a black hole. It shows that only the mass of the central black hole and the densities of the jet affect the length of the jet.

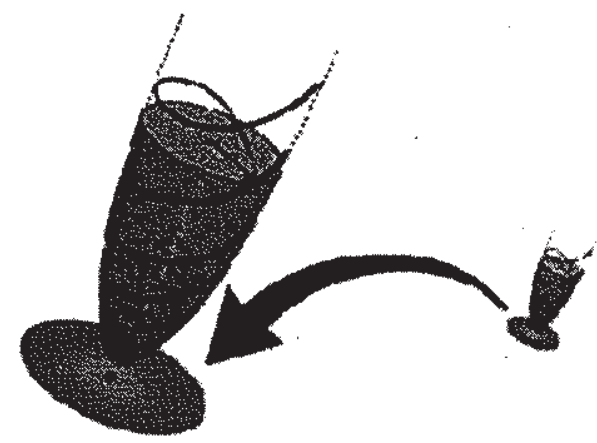

Fig 1.3: Ambient density of the jet [8]

The scaling of the length of the jet with the mass of the black hole is shown in the following table

Table 1.1

\begin{tabular}{|c|c|}
\hline$M\left(M_{\Theta}\right)$ & $/$ (parsec) \\
\hline $10^{2}$ & 2 \\
\hline $10^{4}$ & 20 \\
\hline $10^{6}$ & 200 \\
\hline $10^{8}$ & 2000 \\
\hline
\end{tabular}

The behaviour of the length of the jet as a function of the mass of the black hole is shown in the following figure based on equation (1.4).

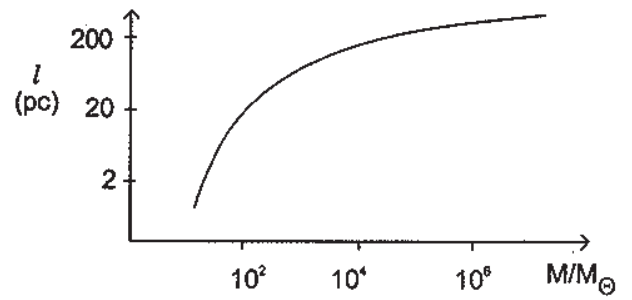

Fig 1.4: Length of the jet with $\mathrm{BH}$ mass 
In the above discussion we considered the variation of the length of the jet with the mass of the black hole. For a given black hole, the length of the jet depends on the density of the particles emitted out along the jet. From our earlier discussion we have,

$$
I=\left(\frac{3 G M^{2}}{\pi c^{2}(\tan 5)^{2}}\right)^{1 / 4} \frac{1}{\rho^{1 / 4}}
$$

For a 30 solar mass black hole, with number density of $n=10^{20}$, the length of the jet is of the order of $1=10^{10} \mathrm{~m}$.

For a billion solar mass black holes, the variation of the length of the jet with respect to the number density of particles in the jet is shown in the following table.

\section{Table 1.2}

\begin{tabular}{|c|c|}
\hline Number density, $n\left(\mathrm{~m}^{3}\right)$ & Length of the jet, $I$ \\
\hline $10^{3}$ & $1 \mathrm{kpc}$ \\
\hline $10^{5}$ & $300 \mathrm{pc}$ \\
\hline $10^{7}$ & $100 \mathrm{pc}$ \\
\hline $10^{20}$ & $0.01 \mathrm{pc}$ \\
\hline
\end{tabular}

\section{Conclusion}

In this paper we have discussed the spinning black hole and its effect in producing the highly collimated, high-energy jets. The results obtained from our calculations are in direct conformation with the observations. In the bibliography we have listed few books for further reading on the subject.

\section{Bibliography} 1. Harry L Shipman, Black Holes, Quasars and the Universe, Houghton Mifflin and Company
(1976)

2. William J Kaufmann III, Black Holes and Warped Space-time, W H Freeman and Co. (1979)

3. Ian Moss, Quantum Theory, Black Holes and Inflation, John Wiley (1996) 
4. J Narliker, Violent Phenomena in the Universe, Oxford Univ. Press (1982)

5. Lian Nicholson, Gravity, Black Holes and the Universe, David and Charles Publication (1981)

6. http://scienceworld.wolfram.com

7. http://chandra.harvard.edu/xroy_sources/blackholes.html

8. http://cosmology.berkeley.edu

9. http://antwrp.gsfc.nasa.gov/htmltest/rin_bht.html 